Pacific Journal of Mathematic 


\title{
A COUNTER-EXAMPLE TO A FIXED POINT CONJECTURE
}

\author{
EARL J. TAFT
}

Let $A$ be a finite-dimentional commutative Jordan algebra over a field $F$ of characteristic zero. Then we may write $A=S+N$, $S$ a semisimple subalgebra (Wedderburn factor), $N$ the radical of $A,[5],[6]$. If $G$ is a completely reducible group of automorphisms of $A$, then we may choose $S$ to be invariant under $G$, [4]. If $G$ is finite, then we showed in [10] that any two such $G$-invariant $S$ were conjugate via an automorphism $\sigma$ of $A$ which centralizes $G$ and which is a product of exponentials of nilpotent inner derivations of $A$ of the form $\sum\left[R_{a_{i}}, R_{x_{i}}\right], x_{i}$ in $N, a_{i}$ in $A$, where $R_{a}$ is multiplication by $a$ in $A$. It was conjectured in [10] that the various elements $x_{i}$ and $a_{i}$ which occur in the formulation of $\sigma$ could be chosen as fixed points of $G$. This conjecture was based on analogous fixed point results proved for associative and Lie algebras, [7], [8], [9]. However, this conjecture is false, and we present in this note a simple counter-example.

We consider three-by-three matrices over $F$. Denoting by $e_{i j}$ the usual matrix units, set $e=e_{11}+e_{22}, f=e_{33}$ and $x=e_{31}$. Consider the Jordan algebra $A$ with basis $e, f, x$ and multiplication table

\begin{tabular}{c|c|c|c|}
\hline$e$ & $f$ & $x$ \\
\hline$e$ & $2 e$ & 0 & $x$ \\
\hline$f$ & 0 & $2 f$ & $x$ \\
\hline$x$ & $x$ & $x$ & 0 \\
\hline
\end{tabular}

Clearly $A$ has a one-dimensional radical $N=F x$, and $S(0)=$ $F e+F f$ is a Wedderburn factor of $A$. By [2], all Wedderburn factors are isomorphic, so are spanned by two orthogonal idempotents. The only idempotents (nonzero) of $A$ are $(e / 2)+\alpha x,(f / 2)+\beta x, \alpha, \beta$ in $F$. The only pairs of orthogonal idempotents are $(e / 2)+\alpha x,(f / 2)-\alpha x$, $\alpha$ in $F$. Hence the Wedderburn factors of $A$ are of the form $S(\alpha)=$ $F(e+\alpha x)+F(f-\alpha x)$, and clearly $\alpha \rightarrow S(\alpha)$ is one-to-one.

A has two types of automorphisms, as can be seen by a direct check. The first type $A(\delta, \pi), \delta, \pi$ in $F, \pi \neq 0$, is given by: 


$$
A(\delta, \pi)\left\{\begin{array}{l}
e \rightarrow f+\delta x \\
f \rightarrow e-\delta x \\
x \rightarrow \pi x
\end{array}\right.
$$

The second type $B(\delta, \pi), \delta, \pi$ in $F, \pi \neq 0$, is given by:

$$
B(\delta, \pi)\left\{\begin{array}{l}
e \rightarrow e+\delta x \\
f \rightarrow f-\delta x \\
x \rightarrow \pi x
\end{array}\right.
$$

A calculation shows that $S(\alpha) B(\delta, \pi)=S(\alpha \pi+\delta)$, so that if $\pi \neq 1$, $S\left((1-\pi)^{-1} \delta\right)$ is the only $B(\delta, \pi)$-invariant Wedderburn factor of $A$. If $\delta \neq 0$, then $B(\delta, 1)$ fixes no Wedderburn factor, and $B(0,1)=I$, the identity mapping of $A$.

Turning to $A(\delta, \pi)$, we have that $S(\alpha) A(\delta, \pi)=S(-\alpha \pi-\delta)$. Hence if $\pi \neq-1, S\left(-\delta(1+\pi)^{-1}\right)$ is the only $A(\delta, \pi)$-invariant Wedderburn factor of $A$. If $\delta \neq 0$, then $A(\delta,-1)$ fixes no Wedderburn factor, but $A(0,-1)$ fixes all Wedderburn factors $S(\alpha)$. Let $G$ be the group of order two generated by $A(0,-1)$ :

$$
A(0,-1)\left\{\begin{array}{l}
e \rightarrow f \\
f \rightarrow e \\
x \rightarrow-x
\end{array} .\right.
$$

Note that $e-f$ and $x$ are eigenvectors for the eigenvalue -1 of $A(0,-1)$, so that $F(e+f)$ is the fixed point space of $G . R_{e+f}=2 I$, and $N$ has no nonzero fixed points under $G$, which disproves the conjecture.

In checking the result of [10] in this example, let $D=\left[R_{e-f}, R_{x}\right]=$ $R_{e-f} R_{x}-R_{x} R_{e-f}$. Then one can check that

$$
\sigma=\exp \left(\left(\frac{\beta-\alpha}{2}\right) D\right)=I+\frac{\beta-\alpha}{2} D
$$

will map $S(\alpha)$ onto $S(\beta)$ for any $\alpha, \beta$ in $F$. Since $e-f$ and $x$ are in the -1 - eigenspace of $A(0,-1)$, the rule $g^{-1} R_{a} g=R_{a g}$ for $a$ in $A, g$ an automorphism of $A$, shows that $D$ commutes with $A(0,-1)$, so that $\sigma$ centralizes $G$. This leads to the more complicated conjecture that one can formulate $\sigma$ in terms of inner derivations $\left[R_{a}, R_{x}\right], a$ in $A, x$ in $N$, such that for any $g$ in $G, a$ and $x$ are eigenvectors of $g$ corresponding to eigenvalues $\alpha(g)$ and $\beta(g)$ respectively, such that $\alpha(g) \beta(g)=1$. Such a $\sigma$ will centralize $G$. We also note that this conjecture and the fixed point conjecture are still open for alternative algebras (see [10] for a precise formulation), although the fixed point conjecture now seems unlikely for alternative algebras, in view of the 
above counter-example for Jordan algebras, due to the close relation between alternative and Jordan algebras, [3]. We also remark that for completely reducible $G$, the existence of a $\sigma$ centralizing $G$ is still an open question. If $N^{2}=0$, this is trivial (see [10], §5), and the difficulty lies in the case $N^{2} \neq 0$. We also note that if $F$ is any field of characteristic not two, then our example has $A / N$ separable and $N^{2}=0$, in which case the Wedderburn-Malcev properties hold, [1], [2], [6], and any finite group $G$ of order not divisible by the characteristic of $F$ will fix a Wedderburn factor, [6]. So our example also shows that the fixed point conjecture is false for the case $N^{2}=0$, $R / N$ separable.

We conclude with an example of an infinite group $G$ which illustrates the conjecture for completely reducible $G$ that $\sigma$ can be chosen to centralize $G$, in a case where $N^{2} \neq 0$. Again considering three-by-three matrices over $F$, let $e=e_{11}+e_{33}, x=e_{12}, y=e_{23}, z=e_{13}$. Let $A$ be the Jordan algebra with basis $e, x, y, z$ and multiplication table

\begin{tabular}{c|c|c|c|c|}
\hline & $e$ & $x$ & $y$ & $z$ \\
\hline$e$ & $2 e$ & $x$ & $y$ & $2 z$ \\
\hline$x$ & $x$ & 0 & $z$ & 0 \\
\hline$y$ & $y$ & $z$ & 0 & 0 \\
\hline$z$ & $2 z$ & 0 & 0 & 0 \\
\hline
\end{tabular}

Clearly the radical $N$ of $A$ is $N=F x+F y+F z, N^{2}=K z$ and $N^{3}=0$. Clearly $S(0,0)=K e$ is a Wedderburn factor, and if we calculate the elements $f$ for which $f^{2}=2 f$, we find

$$
f=e+\alpha x+\beta y-\alpha \beta z, \alpha, \beta \in F .
$$

Since all Wedderburn factors are isomorphic (we are assuming characteristic zero), the Wedderburn factors are of the form

$$
S(\alpha, \beta)=F(e+\alpha x+\beta y-\alpha \beta z),
$$

and the correspondence $(\alpha, \beta) \rightarrow S(\alpha, \beta)$ is one-to-one on $F \times F$.

Let $\delta \in F, \phi \in F, \phi \neq 0,1$. Let $A(\delta, \phi)$ be the automorphism of $A$ given by:

$$
A(\delta, \phi)\left\{\begin{array}{l}
e \rightarrow e+\delta y \\
x \rightarrow x-\delta z \\
y \rightarrow \phi y \\
z \rightarrow \phi z
\end{array}\right.
$$


$A(\delta, \phi)$ is completely reducible, since $A$ has a basis of eigenvectors $y, z,(1-\phi) e+\delta y,(1-\phi) x-\delta z$, the latter two being fixed points of $A(\delta, \phi)$. One can check that $S(\alpha, \beta) A(\delta, \phi)=S(\alpha, \delta+\beta \phi)$, so that $S\left(\alpha, \delta(1-\phi)^{-1}\right)$ is fixed by $G$, the group generated by $A(\delta, \phi)$, for any $\alpha$ in $F$. For $\alpha, \alpha^{\prime}$ in $F$, set

$$
D=\left(\alpha^{\prime}-\alpha\right)(1-\phi)^{-2}\left[R_{(1-\phi) e+\delta y}, R_{(1-\phi) x-\delta \tilde{z}}\right] \text {. }
$$

Then one can calculate that $\sigma=\exp D=I+D+\left(D^{2} / 2\right)$ carries $S\left(\alpha, \delta(1-\phi)^{-1}\right)$ onto $S\left(\alpha^{\prime}, \delta(1-\phi)^{-1}\right)$, and centralizes $G$ since the elements $(1-\phi) e+\delta y,(1-\phi) x-\delta z$ are fixed points of $A(\delta, \phi)$. Note that if $\phi$ is not a root of unity, then $G$ is an infinite group.

Another automorphism $B(\delta, \tau)$ of $A$, for $\delta, \tau$ in $F, \tau \neq 0$, is given by:

$$
B(\delta, \tau)\left\{\begin{array}{l}
e \rightarrow e-\delta \tau x+\delta y+\delta^{2} \tau z \\
x \rightarrow \tau^{-1} y+\delta z \\
y \rightarrow \tau x-\delta \tau z \\
z \rightarrow z
\end{array} .\right.
$$

$B(\delta, \tau)$ has a three-dimensional fixed point space spanned by $e+\delta y$, $z$ and $\tau x+y$, and an eigenvector $\tau x-y-\delta \tau z$ for the eigenvalue -1 , so that $B(\delta, \tau)$ is completely reducible. Actually $B(\delta, \tau)^{2}=I$, so $G$ here is a group of order two. One calculates that $S(\alpha, \beta) B(\delta, \tau)=$ $S\left(-\delta \tau+\beta \tau, \delta+\alpha \tau^{-1}\right)$. Hence $S\left(\alpha, \delta+\alpha \tau^{-1}\right)$ is $G$-invariant for any $\alpha \in F$. Set $D^{\prime}=\tau^{-1}\left(\alpha^{\prime}-\alpha\right)\left[R_{e+\hat{\jmath} y}, R_{z x+y}\right]$ for $\alpha, \alpha^{\prime} \in F$. Then

$$
\sigma=\exp D^{\prime}=I+D^{\prime}+\frac{\left(D^{\prime}\right)^{2}}{2}
$$

carries $S\left(\alpha, \delta+\alpha \tau^{-1}\right)$ onto $S\left(\alpha^{\prime}, \delta+\alpha^{\prime} \tau^{-1}\right)$, and centralizes $G$ since $e+\delta y$ and $\tau x+y$ are fixed points of $B(\delta, \tau)$. Hence, in this case, the fixed point property holds, although, as we have seen in our first example, it does not hold for every finite group $G$.

\section{REFERENCES}

1. B. Harris, Derivations of Jordan algebras, Pacific. J. Math. 9 (1959), 495-512.

2. N. Jacobson, General representation theory of Jordan algebras, Trans. Amer. Math. Soc. 70 (1951), 509-530.

3. - Structure of alternative and Jordan bimodules, Osaka Math. J. 6 (1954), 1-71.

4. G. D. Mostow, Fully reducible subgroups of algebraic groups, Amer. J. Math. 78 (1956), 200-221.

5. A. J. Penico, The Wedderburn principal theorem for Jordan algebras, Trans. Amer. Math. Soc. 70 (1951), 404-421.

6. E. J. Taft, Invariant Wedderburn factors, Illinois J. Math. 1 (1957), 565-573.

7. - Uniqueness of invariant Wedderburn factors, Illinois, J. Math. 6 (1962), 
353-356.

8. — Invariant Levi factors, Michigan Math. J. 9 (1962), 65-68.

9. - Orthogonal conjugacies in associative and Lie algebras, Trans. Amer. Math. Soc. 113 (1964), 18-29.

10. - Invariant splitting in Jordan and alternative algebras, Pacific J. Math. 15 (1965), 1421-1427.

Received August 2, 1967. Research supported by National Science Foundation Grant GP-7162.

Rutgers, The State University 



\section{PACIFIC JOURNAL OF MATHEMATICS}

\section{EDITORS}

\section{H. ROYDEN}

Stanford University

Stanford, California

\author{
R. R. Phelps \\ University of Washington \\ Seattle, Washington 98105
}

\section{J. DugundJI}

Department of Mathematics University of Southern California Los Angeles, California 90007

\section{RICHARD ARENS}

University of California

Los Angeles, California 90024

\section{ASSOCIATE EDITORS}

\section{E. F. BECKENBACH}

B. H. NEUMANN

F. WOLF

K. YOSIDA

\section{SUPPORTING INSTITUTIONS}

UNIVERSITY OF BRITISH COLUMBIA CALIFORNIA INSTITUTE OF TECHNOLOGY UNIVERSITY OF CALIFORNIA MONTANA STATE UNIVERSITY UNIVERSITY OF NEVADA NEW MEXICO STATE UNIVERSITY OREGON STATE UNIVERSITY UNIVERSITY OF OREGON OSAKA UNIVERSITY UNIVERSITY OF SOUTHERN CALIFORNIA

\author{
STANFORD UNIVERSITY \\ UNIVERSITY OF TOKYO \\ UNIVERSITY OF UTAH \\ WASHINGTON STATE UNIVERSITY \\ UNIVERSITY OF WASHINGTON \\ AMERICAN MATHEMATICAL SOCIETY \\ CHEVRON RESEARCH CORPORATION \\ TRW SYSTEMS
}

NAVAL WEAPONS CENTER

Mathematical papers intended for publication in the Pacific Journal of Mathematics should be in typed form or offset-reproduced, double spaced with large margins. Underline Greek letters in red, German in green, and script in blue. The first paragraph or two must be capable of being used separately as a synopsis of the entire paper. It should not contain references to the bibliography. Manuscripts, in duplicate if possible, may be sent to any one of the four editors. All other communications to the editors should be addressed to the managing editor, Richard Arens, University of California, Los Angeles, California 90024.

Each author of each article receives 50 reprints free of charge; additional copies may be obtained at cost in multiples of 50 .

The Pacific Journal of Mathematics is published monthly. Effective with Volume 16 the price per volume (3 numbers) is $\$ 8.00$; single issues, $\$ 3.00$. Special price for current issues to individual faculty members of supporting institutions and to individual members of the American Mathematical Society: $\$ 4.00$ per volume; single issues $\$ 1.50$. Back numbers are available.

Subscriptions, orders for back numbers, and changes of address should be sent to Pacific Journal of Mathematics, 103 Highland Boulevard, Berkeley 8, California.

Printed at Kokusai Bunken Insatsusha (International Academic Printing Co., Ltd.), 7-17, Fujimi 2-chome, Chiyoda-ku, Tokyo, Japan.

PUBLISHED BY PACIFIC JOURNAL OF MATHEMATICS, A NON-PROFIT CORPORATION

The Supporting Institutions listed above contribute to the cost of publication of this Journal, but they are not owners of publishers and have no responsibility for its content or policies. 


\section{Pacific Journal of Mathematics}

\section{Vol. 27, No. $2 \quad$ February, 1968}

Leonard E. Baum and George Roger Sell, Growth transformations for

functions on manifolds ............................ 211

Henry Gilbert Bray, A note on CLT groups ................... 229

Paul Robert Chernoff, Richard Anthony Rasala and William Charles

Waterhouse, The Stone-Weierstrass theorem for valuable fields....... 233

Douglas Napier Clark, On matrices associated with generalized

interpolation problems ................................

Richard Brian Darst and Euline Irwin Green, On a Radon-Nikodym theorem for finitely additive set functions . ...................... 255

Carl Louis DeVito, A note on Eberlein's theorem..................... 261

P. H. Doyle, III and John Gilbert Hocking, Proving that wild cells exist . . . 265

Leslie C. Glaser, Uncountably many almost polyhedral wild $(k-2)$-cells in

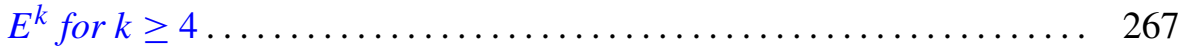

Samuel Irving Goldberg, Totally geodesic hypersurfaces of Kaehler

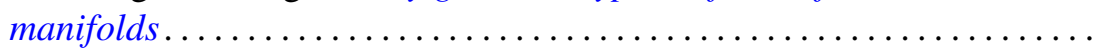

Donald Goldsmith, On the multiplicative properties of arithmetic functions .................................... 283

Jack D. Gray, Local analytic extensions of the resolvent ............ 305

Eugene Carlyle Johnsen, David Lewis Outcalt and Adil Mohamed Yaqub,

Commutativity theorems for nonassociative rings with a finite division ring homomorphic image ....................

André (Piotrowsky) De Korvin, Normal expectations in von Neumann

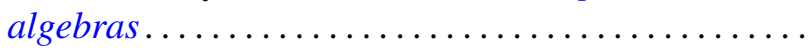

James Donald Kuelbs, A linear transformation theorem for analytic

Feynman integrals..........................

W. Kuich, Quasi-block-stochastic matrices ................... 353

Richard G. Levin, On commutative, nonpotent archimedean

semigroups ............................... 365

James R. McLaughlin, Functions represented by Rademacher series ... . . . 373

Calvin R. Putnam, Singular integrals and positive kernels............ 379

Harold G. Rutherford, II, Characterizing primes in some noncommutative

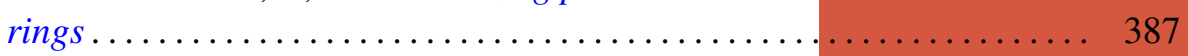

Benjamin L. Schwartz, On interchange graphs................... 393

Satish Shirali, On the Jordan structure of complex Banach *algebras . . . . . 397

Earl J. Taft, A counter-example to a fixed point conjecture............. 405

J. Roger Teller, On abelian pseudo lattice ordered groups ..... 